\title{
Genomewide patterns of variation in genetic diversity are shared among populations, species and higher-order taxa
}

\author{
Nagarjun Vijay ${ }^{1,2}$ (D) | Matthias Weissensteiner ${ }^{1,3}$ | Reto Burri ${ }^{1,4}$ (D) | \\ Takeshi Kawakami $^{1,5} \mid$ Hans Ellegren ${ }^{1}$ (D) | Jochen B. W. Wolf ${ }^{1,3}$
}

${ }^{1}$ Department of Evolutionary Biology and SciLifeLab, Uppsala University, Uppsala, Sweden

${ }^{2}$ Lab of Molecular and Genomic Evolution, Department of Ecology and Evolutionary Biology, College of Literature, Science, and the Arts, University of Michigan, Ann Arbor, MI, USA

${ }^{3}$ Division of Evolutionary Biology, Faculty of Biology, Ludwig-Maximilians-Universität München, Planegg-Martinsried, Germany

${ }^{4}$ Department of Population Ecology, Friedrich Schiller University Jena, Jena, Germany

${ }^{5}$ Department of Animal and Plant Sciences, University of Sheffield, Sheffield, UK

\section{Correspondence}

Nagarjun Vijay and Jochen B. W. Wolf, Department of Evolutionary Biology and SciLifeLab, Uppsala University, Uppsala, Sweden.

Emails: nagarjun.vijay@ebc.uu.se and j.wolf@bio.Imu.de

Funding information

Schweizerischer Nationalfonds zur Förderung der Wissenschaftlichen Forschung, Grant/Award Number: PBLAP3134299, PBLAP3 140171; Swedish Research Council, Grant/Award Number: 621-2010-5553, 2014-6325, 2013-08721; Marie Sklodowska Curie Actions, Grant/ Award Number: 600398; European Research Council, Grant/Award Number: ERCStG336536; Knut and Alice Wallenberg Foundation; Swedish National Infrastructure for Computing

\begin{abstract}
Genomewide screens of genetic variation within and between populations can reveal signatures of selection implicated in adaptation and speciation. Genomic regions with low genetic diversity and elevated differentiation reflective of locally reduced effective population sizes $\left(N_{\mathrm{e}}\right)$ are candidates for barrier loci contributing to population divergence. Yet, such candidate genomic regions need not arise as a result of selection promoting adaptation or advancing reproductive isolation. Linked selection unrelated to lineage-specific adaptation or population divergence can generate comparable signatures. It is challenging to distinguish between these processes, particularly when diverging populations share ancestral genetic variation. In this study, we took a comparative approach using population assemblages from distant clades assessing genomic parallelism of variation in $N_{\mathrm{e}}$. Utilizing population-level polymorphism data from 444 resequenced genomes of three avian clades spanning 50 million years of evolution, we tested whether population genetic summary statistics reflecting genomewide variation in $N_{\mathrm{e}}$ would covary among populations within clades, and importantly, also among clades where lineage sorting has been completed. All statistics including population-scaled recombination rate $(\rho)$, nucleotide diversity $(\pi)$ and measures of genetic differentiation between populations $\left(F_{\mathrm{ST}}\right.$, PBS, $\left.d_{x y}\right)$ were significantly correlated across all phylogenetic distances. Moreover, genomic regions with elevated levels of genetic differentiation were associated with inferred pericentromeric and subtelomeric regions. The phylogenetic stability of diversity landscapes and stable association with genomic features support a role of linked selection not necessarily associated with adaptation and speciation in shaping patterns of genomewide heterogeneity in genetic diversity.
\end{abstract}

\section{KEYWORDS}

background selection, genetic diversity, genetic draft, genetic hitchhiking, linked selection, recombination rate, speciation genetics

\section{1 | INTRODUCTION}

Understanding the processes governing heterogeneity of genomewide diversity has been a long-standing goal in evolutionary genetics (Ellegren \& Galtier, 2016) and is of central importance to adaptation and speciation research (Seehausen et al., 2014; Wolf \& Ellegren, 2017). A plethora of recent studies characterizing genetic variation of diverging natural populations in a taxonomically diverse set of species identified strong heterogeneity in the genomewide distribution of genetic diversity, both within and between populations (e.g., 
in sunflowers (Renaut et al., 2013), monkey flowers (Puzey, Willis, \& Kelly, 2017), stickleback fish (Roesti, Kueng, Moser, \& Berner 2015), rabbits (Carneiro et al., 2014) or birds (Ellegren et al., 2012; Poelstra et al., 2014)). Despite commonality in patterns seen across this wide range of taxa, elucidating the underlying processes remains challenging (Wolf \& Ellegren, 2017).

Regions of reduced genetic diversity generally coinciding with elevated levels of genetic differentiation (Charlesworth, 1998) can be interpreted in the context of adaptation and speciation under conditions of gene flow (Nosil \& Feder, 2013). Building on the idea of a 'genic view of speciation' ( $\mathrm{Wu}, 2001)$, barrier loci experiencing divergent selection contribute to a reduction of gene flow between populations (i.e., reduced effective migration rate $\left(m_{e}\right)$ relative to gross migration rate $(m)$ (Abbott et al., 2013)). However, recombination decouples the locus under divergent selection from neighbouring genetic variation. As a consequence, effective migration rates will not only vary across the genome as a function of the strength of selection ( $s$ ), but also due to recombination rate $(r)$. Effective migration will be most strongly reduced by selection at the causative locus and increases as a function of genetic distance to levels experienced by neutral genetic variation (at equilibrium $m_{e}=m /(1+s / r$ ), (Barton \& Bengtsson, 1986)). Assuming neutrality, empirical information on genomewide migration rate under mutation-drift equilibrium can be obtained from measures of genetic differentiation, usually $F_{\mathrm{ST}} \sim 1 /$ $\left(1+N_{e}(m+\mu)\right)$. Genome scans assaying local levels of genetic differentiation along the genome may additionally allow identifying regions under selection (Lewontin \& Krakauer, 1973). Positive selection will reduce local levels of genetic diversity, and hence $N_{\mathrm{e}}$, resulting in increased levels of $F_{S T}$ (see also (Cruickshank \& Hahn, 2014)). Divergent selection opposing gene flow between populations will further increase regional genetic differentiation by preventing homogenizing admixture (reducing $m_{\mathrm{e}}$ ). Regions of the genome with elevated levels of genetic differentiation and reduced levels of genetic diversity are thus often regarded as candidates for hosting barrier loci subject to divergent selection and refractory to the homogenizing process of gene flow ('speciation islands') (Nosil \& Feder, 2013). Although often framed in the context of ecological speciation (Nosil \& Feder, 2013), barrier loci refer to any genetic element conveying ecological, sexual, pre- or postzygotic reproductive isolation (Wolf, Lindell, \& Backström, 2010). The cumulative effect of multiple barrier loci is eventually expected to transition to genomewide barriers, ultimately promoting speciation (Abbott et al., 2013; Barton, 1983).

However, divergent selection promoting lineage-specific adaptation or reproductive isolation under conditions of gene flow is not the only process introducing heterogeneity in $\mathrm{N}_{\mathrm{e}}$ across the genome. Any form of selection that reduces genetic diversity will result in comparable signatures of genomewide heterogeneity in $N_{e}$. Selection reducing diversity not only at sites under selection, but also at linked neutrally evolving sites, is collectively referred to as linked selection. This includes both positive selection (Smith \& Haigh, 1974) and negative (background) selection (Charlesworth, 1994; Charlesworth, Morgan, \& Charlesworth, 1993). Although these two selective mechanisms are fundamentally different, it is difficult to discern their effect on genetic diversity and differentiation (Stephan, 2010). Linked selection is expected to be most pronounced in regions of low recombination and high target (gene) density and has been shown to significantly affect heterogeneity in levels of genetic diversity across a broad range of organisms (Burri et al., 2015; Cutter \& Payseur, 2013; Nachman \& Payseur, 2012; Slotte, 2014). Genomic regions subject to linked selection are not only depleted of genetic diversity $\left(\theta \sim N_{e} \mu\right)$, but also experience accelerated lineage sorting resulting in increased levels of relative genetic differentiation $\left(F_{\mathrm{ST}}\right)$ (Cruickshank \& Hahn, 2014; Renaut et al., 2013). Relating patterns of genetic variation and differentiation to the underlying process is further complicated by additional intrinsic and extrinsic factors such as mutation rate variation or demographic perturbation (Strasburg et al., 2012).

Several ways forward have been suggested to differentiate between linked selection universally acting in all populations from lineage-specific selection promoting adaptation and speciation. Functional validation of candidate barrier loci flagged during genome scans provides valuable, independent information on the plausibility of divergent selection opposing gene flow in a given population-specific context (Kronforst \& Papa, 2015). Theoretical models provide useful null expectations to compare with empirical patterns (Bank, Ewing, Ferrer-Admettla, \& Foll, Jensen, 2014). Experimental evolution studies (Dettman, Sirjusingh, Kohn, \& Anderson, 2007) or manipulative experiments in natural populations (Soria-Carrasco et al., 2014) allow the link between the nature of selection and genomic patterns of genetic diversity to be studied under controlled conditions. Microlevel comparative population approaches leveraging information from spatiotemporal contrasts between populations ('speciation continuum' (Mallet, Beltrán, Neukirchen, \& Linares 2007; Powell et al., 2013; Seehausen et al., 2014)) help disentangle the effects of linked selection unrelated to speciation (e.g., background selection) from those thought to contribute to reproductive isolation in the face of gene flow (e.g., divergent selection) (Wolf \& Ellegren, 2017). This includes the use of natural hybrids (Barton, 1983; Gompert \& Buerkle, 2011) or crosses generated in the laboratory (Seehausen et al., 2014). Within species and among closely related species, however, a substantial fraction of genetic variation is shared by ancestry, impeding inference.

Here, we propose a macrolevel comparative approach extending comparisons of genomewide diversity beyond closely related taxa to phylogenetically distant clades, where lineage sorting has long been completed. This controls for the effect of shared recent ancestry, recent or ongoing gene flow between clades. Genomic parallelism in patterns of genetic diversity across such large evolutionary distances cannot be explained by processes involving selection on a set of specific genes for each lineage. Instead, it is expected that genomic parallelism is mediated by universal processes shared in syntenic regions with similar genomic properties among clades.

One candidate parameter to affect genetic diversity $\left(\theta \sim 4 N_{\mathrm{e}} \mu\right)$ of syntenic regions similarly among clades is the mutation rate $\mu$, which is known to vary across the genome (Hodgkinson \& EyreWalker, 2011). However, support for a role of mutation rate in modulating the level of genetic variation and differentiation across the 
genome is limited (Cutter \& Payseur, 2013). While some studies found a contribution (Dutoit et al., 2017; Smith \& Eyre-Walker, 2017), genetic diversity is generally only weakly associated with proxies for mutation rate (Cutter \& Payseur, 2013; Vijay et al., 2016). Another parameter that can affect genetic diversity is recombination rate which is reportedly conserved at broadscale between clades (Auton et al., 2012; Burri et al., 2015; Kawakami et al., 2014; Roesti, Hendry, Salzburger, \& Berner, 2012; Singhal et al., 2015; Tine et al., 2014). With little evidence for recombination-associated mutation (and hence $r \sim \mu$ ) (Cutter \& Payseur, 2013), any form of linked selection, where the local reduction in $N_{e}$ through selection is contingent on the rate of local recombination, is thus a prime candidate for explaining shared heterogeneity in genetic variation among clades (Cutter \& Payseur, 2013).

A macrolevel comparative perspective on genomewide variation of genetic diversity is implicit, though not the main focus, of recent work by Van Doren et al. (2017) and Dutoit et al. (2017) comparing summary statistics of genetic diversity between stonechats and flycatchers and between flycatchers and crows, respectively. Here, we assess the contribution of linked selection in shaping genomewide landscapes of genetic diversity and differentiation across a wide range of evolutionary time scale ranging from few thousand to approximately 50 million years of evolution. Given the global conservation of recombination landscape for tens of millions of years among avian lineages (Singhal et al., 2015), it is expected that linked selection mediated by recombination constitutes an important component for the concerted evolution of heterogeneity in genomewide diversity. Note that linked selection resulting in genomic parallelism between clades includes background selection as well as positive selection acting repeatedly on orthologous loci among clades. We, therefore, predict that summary statistics reflective of $N_{e}$ not only covary among populations of closely related taxa, but are also correlated among clades. Moreover, assuming karyotypic stability, we would expect genomic regions with locally reduced $N_{e}$ by linked selection to be stably associated with chromosomal features of suppressed recombination such as pericentromeric or subtelomeric regions.

To empirically address this expectation, we used publicly available genome resequencing data from several populations or (sub)-species of three distantly related clades of avian species complexes - Darwin's finches, Ficedula flycatchers and Corvus crows (Table S1) - with split times beyond the expected time for complete lineage sorting (Fig. S1). For each population and species comparison within clades, we quantified a set of genetic summary statistics in syntenic windows of $50 \mathrm{~kb}$ in size. Summary statistics were chosen to be reflective of the local effective population size $\left(N_{e}\right)$ of a genomic region: population-scaled recombination rate $\rho\left(\sim N_{\mathrm{e}} r\right)$, nucleotide diversity $\pi\left(\sim N_{\mathrm{e}} \mu\right)$, genetic differentiation expressed as $F_{\mathrm{ST}}(\sim 1 /$ $\left(1+N_{\mathrm{e}}(m+\mu)\right)$ (where mutation rate $\mu$ can generally be neglected if migration rate $m \gg \mu$ ), the related population branch statistic (PBS) accounting for nonindependence of population comparisons, and $d_{x y}$ $\left(\sim N_{\mathrm{e}} \mu+\mu \mathrm{t}\right)$ reflecting the average number of nucleotide substitutions between populations. The only parameter shared by these statistics is $N_{\mathrm{e}}$; hence, covariation of all statistics in syntenic regions would indicate selection affecting local $N_{e}$ alike in the investigated populations.

\section{2 | MATERIALS AND METHODS}

\section{1 | Clades}

We chose populations and (sub)-species from three phylogenetically divergent clades: Darwin's finches of the genera Geospiza, Certhidea and Platyspiza., flycatchers of the genus Ficedula (F. albicollis, F. hypoleuca, F. semitorquata and F. speculigera) and crows of the genus Corvus including the American crow C. brachyrhynchos and several taxa from the Corvus (corone) spp. species complex (Vijay et al., 2016). Functionally annotated genome assemblies with high sequence contiguity are available for one representative each of Ficedula flycatchers (F. albicollis, genome size: 1.13, scaffold/contig $\mathrm{N} 50=6.5 \mathrm{Mb} / 410 \mathrm{~kb}$, National Center for Biotechnology Information (NCBI) Accession No: GCA_000247815.2; (Ellegren et al., 2012); new chromosome build (Kawakami et al., 2014)) and for one hooded crow specimen (Corvus (corone) cornix, genome size: $1.04 \mathrm{~Gb}$, scaffold/contig $\mathrm{N} 50=16.4 \mathrm{Mb} / 94 \mathrm{~kb}, \mathrm{NCBI}$ Accession no: GCA 000738735.1; (Poelstra et al., 2014; Poelstra, Vijay, Hoeppner, \& Wolf, 2015)). The assembly of the medium ground finch $G$. fortis is of comparable size $(1.07 \mathrm{~Gb})$ and the least contiguous among the three both at the scaffold and contig level (scaffold/contig $\mathrm{N} 50=5.3 \mathrm{Mb} / 30 \mathrm{~kb}, \quad \mathrm{NCBI}$ Accession no: GCA_000277835.1; (Rands et al., 2013)).

In all three clades, it has been suggested that shared genetic variation between (sub)-species within clades resulted from incomplete lineage sorting of ancestral polymorphisms, regardless of whether populations were connected by recent gene flow or not (Burri et al., 2015; Lamichhaney et al., 2015; Vijay et al., 2016). However, shared polymorphism is highly unlikely among clades because of their phylogenetic distance. Phylogenetic relationships and divergence time estimates between representatives of all three clades and zebra finch (Taenopygia guttata) as shown in Figure 1 have been extracted as the consensus of 10,000 phylogenetic reconstructions from Jetz, Thomas, Joy, Hartmann, and Mooers (2012) and Jetz et al. (2014) using the tree of 6670 taxa with sequence information by Ericson et al. (2006) as backbone (http://birdtree.org/). This places the separation between Corvoidea (crows) and Passerida (Darwin's finches and flycatchers) at over 50 million years. Assuming a range in generation time between 6 years for hooded crows (Vijay et al., 2016), 5 years for Darwin's finches (Grant \& Grant, 1992) and 2 years for flycatchers (Brommer, Gustafsson, Pietiäinen, \& Merilä, 2004), this corresponds to at least 8-25 million generations. With an estimated long-term $\mathrm{N}_{\mathrm{e}}$ of 200,000 for flycatchers and crows (NadachowskaBrzyska et al., 2013; Vijay et al., 2016; Wolf, Bayer, et al., 2010; Wolf, Lindell, et al., 2010) and considerably less for Darwin's finches $\left(N_{\mathrm{e}}=6,000\right.$ to 60,000 (Lamichhaney et al., 2015)), this yields a minimum range of $40-125 \mathrm{~N}_{\mathrm{e}}$ generations as time to the most common ancestor. This is clearly beyond the expected time for complete 


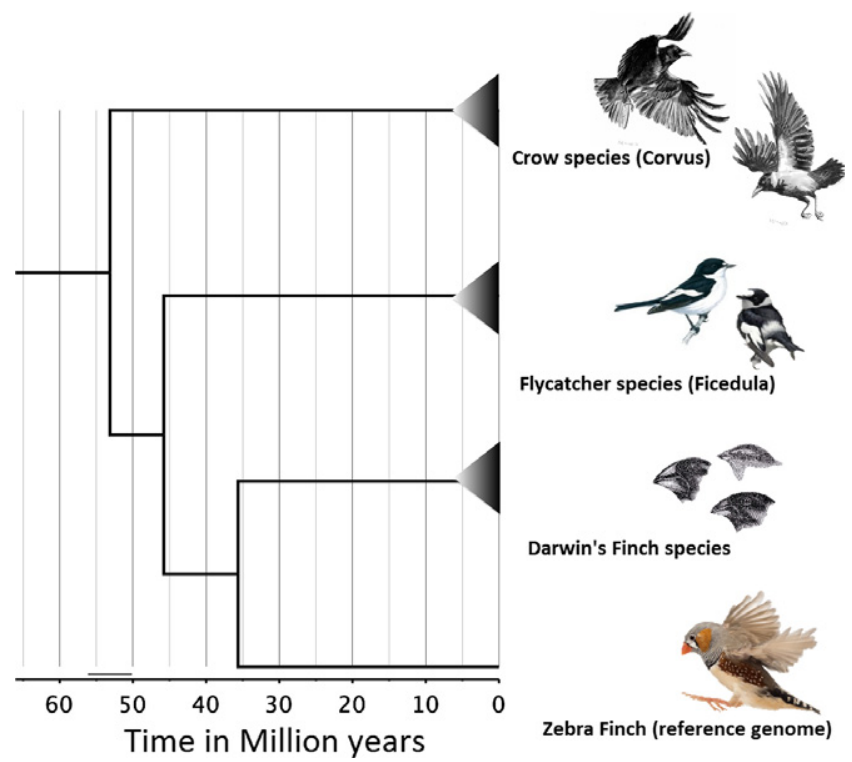

FIGURE 1 Study design. Dated phylogenetic reconstruction of all clades used in this study. Note that for each focal taxon (crows, flycatchers and Darwin's finches), a large number of individuals from several populations and subspecies have been used comprising 120 Darwin's finch genomes (Lamichhaney et al., 2015), 200 genomes from Ficedula flycatchers (Burri et al., 2015) and 124 genomes from crow of the genus Corvus (Vijay et al., 2016) [Colour figure can be viewed at wileyonlinelibrary.com]

lineage sorting (9-12 $N_{e}$ generations; (Hudson \& Coyne, 2002)). Clades are thus not expected to share ancestral polymorphism. The same consideration holds for the split between flycatcher and Darwin's finches assuming approximately 45 million years of divergence (Figure 1). Even assuming an earlier, minimal age estimate of the split between Corvoidea and Passerida in the order of 25 million years ago (Jarvis et al., 2014; Prum et al., 2015; Jønsson et al. 2016) and a split between flycatchers and finches at 19 million years (Singhal et al., 2015) gives split times beyond $12 \mathrm{~N}_{\mathrm{e}}$ generations suggesting complete lineage sorting for neutral genetic variation.

\section{2 | Establishing homology among genomes}

Homologous regions between genomes were identified in order to quantify the degree to which genetic diversity, recombination and genetic differentiation landscapes are conserved between species. To ensure comparability across all three clades in the most efficient way, we chose to lift-over coordinates of 50-kb nonoverlapping windows from the genomes to the independent, well maintained highquality zebra finch reference genome (Hubbard et al., 2002). Liftover is the process of transferring the positions along one genome to another genome based on whole-genome alignments. This approach assumes a high degree of synteny among species, which is justified given the evolutionary stasis of chromosomal organization in birds across more than 100 million years of evolution (Ellegren, 2010). Performing a base by base lift-over can lead to partial loss of regions within a window as well as merging of nonadjacent windows.
While sequencing reads of one species can be mapped to the genome of another species to identify variants, this strategy cannot be confidently extended beyond 5-15\% sequence divergence without introducing read mapping bias (Shafer et al., 2016; Vijay, Poelstra, Künstner, \& Wolf, 2013). To avoid such errors, we estimated the statistics for each species in windows prior to the lift-over. Converting the coordinates of genomes from multiple different species into one single coordinate system allows for straightforward comparison of all statistics derived from the original polymorphism data (in variant call format or vcf).

Whole-genome alignments between species can be represented in the form of chain files that record the links between orthologous regions of the genome. We downloaded chain files from the UCSC website (https://genome.ucsc.edu/) to transfer the coordinates in bed format from flycatcher and Darwin's Finch genomes onto the zebra finch genome using the program liftOver (Kuhn et al., 2007). For the crow genome where no chain files were available, we first aligned the crow genome to the flycatcher genome using LASTZ (Harris, 2007) to obtain a .psl file which was subsequently converted to a chain file using JCVI utility libraries (Tang, Li, \& Krishnakumar, 2015). This chain file was then used to transfer the crow coordinates to zebra finch coordinates (via flycatcher) using the liftOver utility (Hinrichs et al., 2006).

Orthology could be established for a large proportion of the original genomes. Depending on parameter settings, controlling stringency ('minmatch') and cohesion ('minblocks') per cent recovery ranged from as little as $13 \%$ to over $90 \%$ (Fig. S1, Table S2). To find an optimal combination of parameter values and to validate lift-over quality, we made use of the fact that GC content in orthologous regions of avian genomes is expected to be strongly conserved across long evolutionary distances (Weber, Boussau, Romiguier, Jarvis, \& Ellegren, 2014). We calculated GC content in 50-kb windows from the three different assemblies and compared these values to the GC content at the new, orthologous positions lifted over to the zebra finch genome. Pearson's correlations were high across a broad set of parameter values in all clades ranging from 0.83-0.97. While liftOver is able to transfer the coordinates from the focal genome onto positions along the zebra finch genome, these new positions do not retain the window structure from the original genomes. To be able to compare population genetic summary statistics between species in orthologous windows, we defined 50-kb windows along the zebra finch genome. For each window, we then calculated a mean value across all regions that were lifted over and overlapped a given window. To ensure that this procedure of calculating means did not unduly influence comparability across species, we compared the values of GC content from each of the focal genomes after taking the mean across overlapping regions to the GC content in the zebra finch genomic windows. Although correlation coefficients were lower than those seen directly after liftOver, they still exceeded 0.78, 0.82, 0.82 for Darwin's finch, flycatcher and crow, respectively, across a broad 'minmatch' and 'minblock' parameter space (Fig. S1, Table S2). The high correlation of GC content across the liftOver steps suggests that the lift-over procedure of moving the windows 
from one genome assembly to another was reliable at the window size being evaluated. Finally, an optimal combination of stringency, cohesion and per cent recovery was chosen on the basis of the (visually inferred) inflection point of the relationship between GC correlation and recovery (Fig. S1).

It could be seen that certain regions of the genome were systematically more susceptible to drop out during liftOver than others for all clades (Fig. S2). In particular, regions located on scaffolds that have not been linked to any specific chromosome and those that have not been placed at a particular position along a chromosome were more difficult to lift-over than other regions of the genome. Hence, for the purpose of this study, we have excluded these regions in all subsequent analyses. To ensure that liftOver did not introduce a bias in the regions being analysed, we compared the GC content distribution of the regions that could be lifted over at different values of the "minmatch" parameter (Fig. S3). No clear evidence of bias with regard to GC content of the successfully lifted over regions emerged.

\subsection{Data sets}

We compiled the following publicly available population resequencing data sets for the three clades (Table S1). Populations with less than three individuals were excluded in all species.

1. Crows in the genus Corvus (124 genomes resequenced, 55 population comparisons within and between two focal species, the American crow C. brachyrhynchos and various (sub)-species and populations within the $C$. (corone) spp. complex). Population genetic summary statistics including genetic diversity $(\pi)$, population recombination rate $(\rho)$, genetic differentiation $\left(F_{\mathrm{ST}}, \mathrm{PBS}, d_{\mathrm{xy}}\right)$ across the European crow hybrid zone have been characterized using high coverage whole-genome resequencing data of 60 individuals samples in a $2 \times 2$ population design between carrion crows (Corvus (corone) corone) and hooded crows (C. (c.) cornix) (Poelstra et al., 2014). This study has been followed by a broader sampling regime with a total of 118 crows from the Corvus (c.) spp. species complex including a parallel hybrid zone in Russia between C. (c.) cornix and C. (c.) orientalis, a contact zone between the latter and $C$. (c.) pectoralis and numerous other allopatric populations (Vijay et al., 2016). The system is relatively young such that $12 \%$ of segregating genetic variation has been estimated to be shared between Eurasian and American crows (C. brachyrhynchos) (Vijay et al., 2016) which split at approximately 3 million years ago (Jønsson et al. 2016). $F_{\mathrm{ST}}$ and $d_{\mathrm{xy}}$ ranged from 0.016-0.486 and $0.0015-0.0018$, respectively. A broad range in $\pi(0.0010-0.0033)$ and Tajima's D ( 0.5895 to -1.974$)$ suggests perturbation by population-specific demographic histories.

2. Ficedula flycatchers (200 genomes resequenced with 30 population comparisons across the 4 focal species F. albicollis, F. hypoleuca, F. semitorquata and F. speculigera and two outgroup species F. parva and F. hyperythra). Species diverged approximately 2 million years ago and populations differ slightly in genomewide levels of differentiation ( $\pi$ : 0.0029-0.0039). A total of 30 population comparisons within and across species provide a broad contrast across a spectrum of genomewide differentiation ( $F_{\mathrm{ST}}: 0.012-0.981$ and $d_{\mathrm{xy}}: 0.0031-0.0050$ ) (see (Burri et al., 2015)).

3. Darwin's finches (120 genomes resequenced, 44 population comparisons across the six focal species Geospiza conirostris, Geospiza difficilis, Camarhynchus pallidus, Certhidea fusca, Certhidea olivacea and Pinaroloxias inornata). The differentiation landscape of Darwin's finches has been studied using whole-genome resequencing data and has been instrumental in the identification of adaptive loci associated with beak shape evolution (Lamichhaney et al., 2015). This set of populations across several species differs fourfold in genomewide levels of diversity ( $\pi$ : 0.0003-0.0012, see (Lamichhaney et al., 2015)). Species are estimated to share common ancestry $~ 1.5$ million years ago, yielding 44 population comparisons ranging across a broad spectrum of genomewide differentiation ( $\left.F_{\mathrm{ST}}: 0.192-0.897\right)$ and divergence $\left(d_{\mathrm{xy}}: 0.0022-0.0047\right)$.

\section{4 $\quad$ Genetic diversity data}

In all three study systems, segregating genetic variation and related summary statistics have been characterized in nonoverlapping windows across the genome using similar strategies based on the Genome Analysis Toolkit GATK (DePristo et al., 2011) (see Table S3 for methodological comparison and consult individual studies for additional details). We used the final set of variant calls from each individual to calculate a set of summary statistics. vcf (Variant Call Format) files were obtained from Lamichhaney et al. (2015) for Darwin's finches, Burri et al. (2015) for flycatchers and Vijay et al. (2016) for crows. Each of the statistics was calculated in 50-kb windows for all scaffolds longer than $50 \mathrm{~kb}$.

\subsection{1 | Population recombination rate $(\rho)$ and nucleotide diversity $(\pi)$}

To generate an estimate of the population-scaled recombination rate in Darwin's finches $\rho$, we followed the approach described in Vijay et al. (2016). In brief, we used LDHeLmet (Chan, Jenkins, \& Song, 2012) on genotype data phased with FASTPHASE (Scheet \& Stephens, 2006). The required mutation matrix was approximated from zebra finch substitution rates following Singhal et al. (2015). Population recombination rate data for crows and flycatchers were estimated using the same approach and were extracted from Vijay et al. (2016) and Kawakami et al. (2017), respectively. Pairwise nucleotide diversity $\pi$ was calculated from the .vcf files using the R package HIERFSTAT. The number of usable invariant sites was identified based on per base pair sequencing coverage of individuals to use only those sites that are covered by at least five reads in more than half of the individuals in each population.

\subsection{2 | Genetic differentiation $\left(F_{\mathrm{ST}}, \mathrm{PBS}, d_{\mathrm{xy}}\right)$}

$F_{\mathrm{ST}}$ was estimated using Weir and Cockerham's estimator based on genotypes from the .vcf files using the procedure implemented in 
the HIERFSTAT package (Goudet, 2005) as the ratio of the average of variance components. To avoid pseudo-replicated population comparisons, we also calculated lineage-specific $F_{\mathrm{ST}}$ in the form of population branch statistics (PBS) using the formula PBS $=\left(\left(-\log \left(1-F_{\text {ST }}(\right.\right.\right.$ Pop1_Pop2 $\left.\left.)\right)\right)+\left(-\log \left(1-F_{\text {ST }}(\right.\right.$ Pop1_Pop3 $\left.\left.)\right)\right)$ $-\left(-\log \left(1-F_{S T}(\right.\right.$ Pop2_Pop3 $\left.\left.\left.)\right)\right)\right) / 2 . d_{x y}$ following the definition by Nei (1987) was estimated with custom scripts on the basis of the $R$ package HIERFSTAT (Poelstra et al., 2014). The number of usable invariant sites for $d_{x y}$ calculation was identified based on per base pair sequencing coverage of individuals to use only those sites that are covered by at least five reads in more than half of the individuals in both populations.

\subsection{3 | Quantifying similarity of genomic landscapes within and among clades}

We used Pearson correlations as a simple means to characterize the degree of covariation in genomewide distribution patterns for a given summary statistic. Correlation coefficients were calculated on the basis of homologous windows within and between clades (see above). For intrapopulation measures $(\rho, \pi)$, we calculated all possible combinations between two populations (with more than three individuals) $i=1 \ldots(n-1)$ and $j=(i+1) \ldots .$. . For interpopulation metrics $\left(F_{\mathrm{ST}}, \mathrm{PBS}, d_{\mathrm{xy}}\right)$, we calculated all possible combinations between population comparisons I (e.g., popA vs. popB), J (e.g., popC vs. popD) except for flycatcher where $F_{S T}$ was only available for 16 populations comparisons (cf. Burri et al., 2015). This yields a distribution of correlation coefficients for each summary statistic (see also (Vijay et al., 2016)). Significance in covariation between populations or population comparisons was attributed if more than $95 \%$ of the distribution were above zero (significant positive correlation) or below zero (significant negative correlation).

\subsubsection{Overlap with centromeres and subtelomeres}

LiftOvers to the zebra finch genome in principle allow associating outlier regions from genome scans (e.g., islands of elevated differentiation) with genomic features such as centromeres or subtelomeres. This approach works under the assumption of karyotype conservation across large evolutionary timescales (Ellegren, 2010). It is conservative in that overlap is only expected if centromere position is conserved between zebra finch and the taxon under consideration. Evolutionary lability of these features, partly expected due to known lineage-specific inversions in zebra finch (Hooper \& Price, 2015; Kawakami et al., 2014; Romanov et al., 2014), would reduce any real correlation (type II error), but is unlikely to introduce spurious correlations (type I error). Twenty-two centromere and 20 subtelomere positions were obtained for zebra finch from Knief and Forstmeier (2016). Candidate centromeric regions were on average $\sim 1 \mathrm{Mb}$ long (mean: $960,100 \mathrm{bp}$; range: $150,000 \mathrm{bp}$ to $5,350,000 \mathrm{bp}$ ), while the subtelomeric regions were shorter (mean: 169,800; range: 50,000 bp to $298,700 \mathrm{bp}$ ). Some of the subtelomeric and (peri)centromeric regions were located at the extreme ends of the chromosomes and orthologous regions could not be identified in the draft assemblies of the crow, flycatcher and Darwin's finch. These regions are either not assembled in the draft genomes, or synteny could not be unambiguously assigned.

Of the 42 regions that have been identified as (peri)centromeric or subtelomeric regions in zebra finch, orthologous regions could be identified for a subset of 38 in the flycatcher (mean recovery, i.e., mean of the fraction of each of the regions mapped: 0.69), 39 in crow (mean recovery: 0.83) and 25 in the Darwin's Finch genome (mean recovery: 0.55). The relatively low recovery in Darwin's finch is most likely owing to the lower quality of its genome, which is more fragmented than the genomes of flycatcher and, particularly, of crow. The subtelomeres of chromosome 5, 13 and 21 could be lifted over in neither crow nor flycatcher genomes suggesting a systematic bias for these regions. To reduce the effect of such bias, we not only looked for overlap of outlier peaks (as defined below) with (peri)centromeric or subtelomeric regions, but also for overlap with increasing distance from the inferred positions of these features in five incremental steps of $10 \mathrm{~kb}$. In the case of random association, no relationship would be expected with distance. In the case of genuine association, significance of the overlap should decrease with distance.

To relate characteristics of the genomic differentiation landscape to chromosomal features, we proceeded as follows. For each taxon, we chose two independent population comparisons with the highest genomewide average $F_{\mathrm{ST}}$ values. This strategy is owing to the fact that clear 'background peaks' caused by shared linked selection only start crystallizing at an advanced level of population divergence (Burri et al., 2015; Vijay et al., 2016). This is theoretically expected and has been shown in crows where an increase in genomewide $F_{S T}$ is accompanied by an increase in autocorrelation between windows, peak overlap and the degree of covariation in differentiation landscapes (Vijay et al., 2016). Population pairs used and their corresponding differentiation statistics are shown in Table S4. We then used positions along the zebra finch genome to calculate the per cent of (peri)centromeric and subtelomeric regions that overlapped with differentiation outliers (Table S5). To check whether the per cent of overlap we observed was more than that expected by chance, we permuted the positions of centromeres and subtelomeres within each chromosome 1000 times using the shuffle option in bedtools (Quinlan \& Hall, 2010) and calculated the per cent of overlap that was expected by chance alone. A significant association is inferred at type I error levels of $0.05 / 0.01$ if the test statistic derived from the empirical centromere/subtelomere distribution exceeded a maximum of 49/ 0 -times by test statistics derived from the permuted distributions.

\section{RESULTS}

\section{1 | Covariation within clades (microlevel)}

Previous studies in flycatcher (Burri et al., 2015; Kawakami et al., 2017) and crow (Vijay et al., 2016) have shown that populationscaled recombination rate $(\rho)$, nucleotide diversity $(\pi)$ and measures of genetic differentiation $\left(F_{\mathrm{ST}}\right.$, PBS and $d_{\mathrm{xy}}$ ) were significantly 
correlated between population (comparisons) within each clade. Extending the population comparison of $\rho, \pi, F_{S T}$, PBS and $d_{x y}$ to the Darwin's finch complex corroborates the generality of this finding. Genomewide patterns of these summary statistics summarized in Figure 2 and Table S6 were positively correlated among all populations in each of the three clades. For $\rho$, correlation coefficients were highest in flycatchers (mean $r=.43$ ), followed by Darwin's finches $(r=.27)$ and crows $(r=.19)$. Nucleotide diversity $\pi$ showed strongest covariation in flycatchers $(r=.95)$, followed by crows $(r=.70)$ and Darwin's Finches $(r=.49)$. Correlation of $\mathrm{F}_{\mathrm{ST}}$ was consistently positive between all population pairs in Darwin's finches $(r=.46)$, flycatchers (mean $r=.42$ ) and crows $(r=.36)$. The correlation for PBS was even stronger than $F_{\mathrm{ST}}(r=.64$ in Darwin's finches, $r=.46$ in flycatchers and $r=.42$ in crows). $d_{x y}$ showed significantly positive correlations between pairs of populations within each clade with mean correlation coefficients of $.72, .85$ and .94 in flycatchers, crows and Darwin's finches, respectively. Importantly, $d_{x y}$ was negatively correlated with $F_{\mathrm{ST}}$ (mean range $r=-.45$ to -.19 ). This is predicted by long-term linked selection (acting already in the ancestor) and is opposed to the expectation for divergent selection in the face of gene flow (Cruickshank \& Hahn, 2014; Nachman \& Payseur, 2012).

\subsection{Covariation across clades (macrolevel)}

Next, we investigated whether the summary statistics indicative of local $N_{e}$ used in the intraclade comparisons also covaried in syntenic regions between clades. Although effect sizes were lower, correlations were consistently positive for all summary statistics (Figure 2b, Table S7). Mean Pearson's correlation coefficient in the population-scaled recombination rate $(\rho)$ ranged from 0.099 (crow vs. flycatcher) to 0.172 (flycatcher vs. Darwin's finch) and for nucleotide diversity $(\pi)$ from 0.082 (flycatcher vs. Darwin's finch) to 0.271 (crow vs. flycatcher). Patterns of genetic differentiation were also similar between clades with $F_{\mathrm{ST}}$ ranging from 0.115
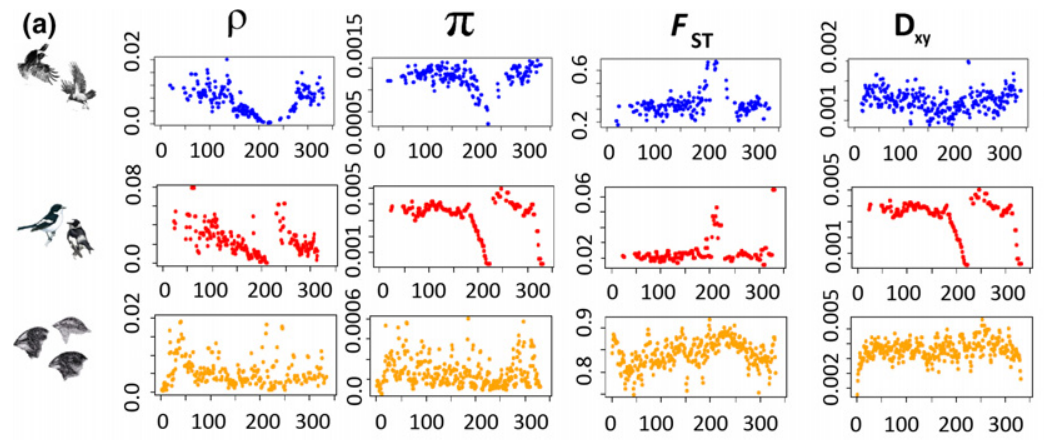

(1)

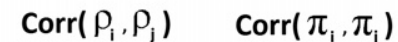

$\operatorname{Corr}\left(\boldsymbol{F}_{\mathrm{sT}}, \boldsymbol{F}_{\mathrm{sT}}\right)$

$\operatorname{Corr}\left(D_{x y I}, D_{x y J}\right)$
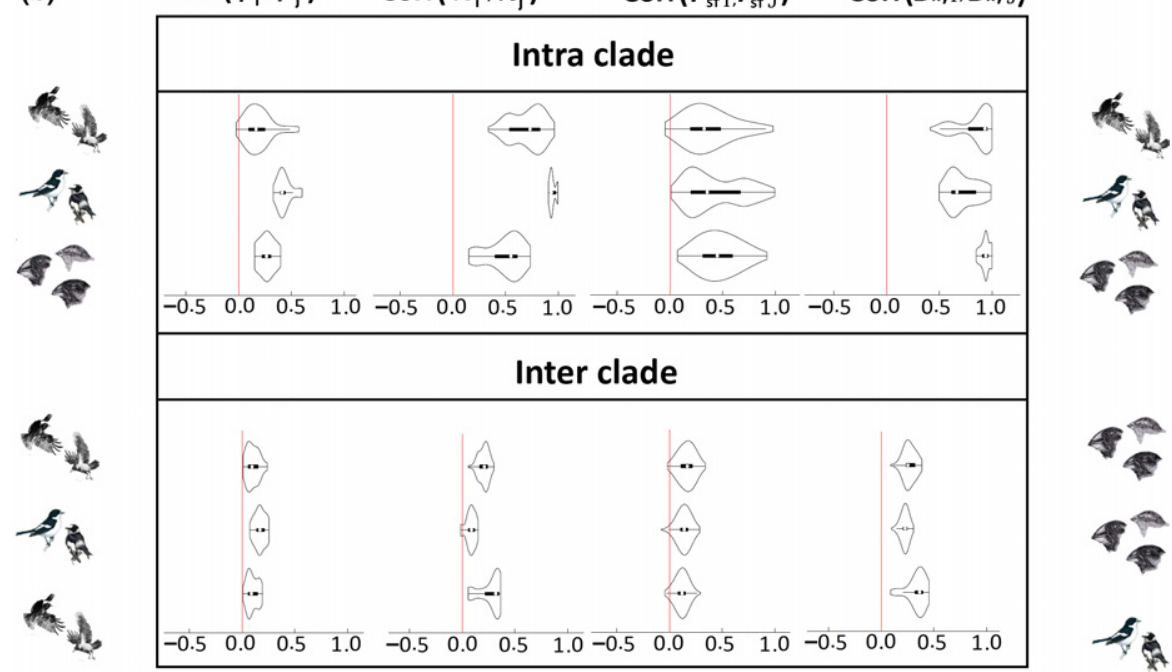

FIGURE 2 Covariation of population genetic summary statistics within and among clades. (a) Genomewide landscapes of four summary statistics are compared within and between clades. Depicted is an example showing the population recombination rate ( $\rho$ ), nucleotide diversity $(\pi)$, genetic differentiation $\left(F_{S T}\right.$ and dxy) along chromosome 13 of zebra finch. The $x$-axis is scaled in units of 50-kb windows. (b) Distribution of correlation coefficients (Pearson's $r$ ) shown as violin plots for population summary statistics characterizing variation within ( $\rho, \pi$ ) and between populations $\left(\mathrm{F}_{\mathrm{ST}}, \mathrm{dxy}\right)$. Correlations are first shown for population comparisons within each of the three clades (intraclade). Subscripts $\mathrm{i}, \mathrm{j}$ symbolize all possible combinations of correlations between two populations $i=1 \ldots(n-1)$ and $j=(i+1) \ldots . . n$ for within-populations measures; capital letters I, J symbolize interpopulation statistics. Correlations exclude pseudo-replicated population comparisons. Similarly, within- and between-population measures were compared among all three clades (interclade), as illustrated by the bird images. In case of no association, a normal distribution centred around null would be expected [Colour figure can be viewed at wileyonlinelibrary.com] 
(crow vs. flycatcher) to 0.163 (crow vs. Darwin's finch) and PBS ranging from 0.185 (crow vs. Darwin's finch) to 0.231 (flycatcher vs Darwin's finch). $d_{x y}$ showed the highest interclade correlations ranging from 0.224 (flycatcher vs. Darwin's finch) to 0.342 (crow vs. flycatcher). As in the microlevel comparisons, $d_{\mathrm{xy}}$ and $F_{\mathrm{ST}}$ were negatively correlated among clades (mean range $r=-.21$ to -.16 ). The strength of correlation in all of these summary statistics was not systematically associated with divergence time representing 50 million years of independent evolution (Figure 2b, Table S7, Fig. S4).

\subsection{Overlap with structural genomic features}

We next sought to investigate the potential impact of structural genomic features where the effect of linked selection might be particularly pronounced. We evaluated whether regions of highly elevated differentiation were associated with regions of suppressed recombination adjacent to pericentromeric and subtelomeric regions as predicted from the location of such regions in zebra finch (karyotype data are not available for both crow and collared flycatcher; Figure 3a). For each clade, we focused on the two most divergent population/species comparisons (Burri et al., 2015; Vijay et al., 2016). In all three clades, the overlap was significantly larger than expected by chance in at least one comparison of each species (percentage of overlap in flycatchers: $58.53 \%$ and $60.98 \%$, crows: 21.95\% and 31.7\%, Darwin's finches: $14.63 \%$ and $29.27 \%$ ) (Figure $3 b)$. When regions next to pericentromeric and subtelomeric regions were considered separately, there was a significant association for subtelomeric regions in all three clades (Fig. S5), whereas the association for regions next to centromeres was significant only in flycatcher (Fig. S6).

\section{DISCUSSION}

In this study, we quantified genomewide patterns of genetic diversity within and between multiple populations for each of three phylogenetically distant avian clades with split times beyond the expected time for complete lineage sorting. We asked the question whether these 'landscapes of genetic diversity' covaried across microevolutionary timescales among populations within clades and across macroevolutionary timescales among clades.

As previously reported, genomewide heterogeneity in genetic variation captured by population genetic statistics reflective of local $\mathrm{Ne}_{\mathrm{e}}$ covaried among populations within clades. Studies in sunflowers (Renaut et al., 2013) stonechats (Van Doren et al., 2017), crows (Vijay et al., 2016) and flycatchers (Burri et al., 2015) similarly reported that landscapes of variation in genetic diversity were correlated among populations and closely related species differing in divergence time and the level of gene flow. An explanation for the correlated pattern of diversity, therefore, requires a mechanism universally affecting all populations. Variation in the strength of linked selection mediated by local levels of recombination rate shared among populations has been suggested as a primary force. In flycatchers, for example, where pedigree-based recombination rate data are available, linked selection serves an explanation for genomic parallelism among populations and species without the need to invoke population-specific adaptation and context-dependent selection in the face of gene flow (Burri et al., 2015). While mutation rate may contribute in shaping genomewide variation in genetic diversity, linked selection appears to be the dominant mechanism (Dutoit et al., 2017).

The present study adds a macroevolutionary, comparative axis providing evidence for linked selection at syntenic regions across large phylogenetic distances where any contribution of shared
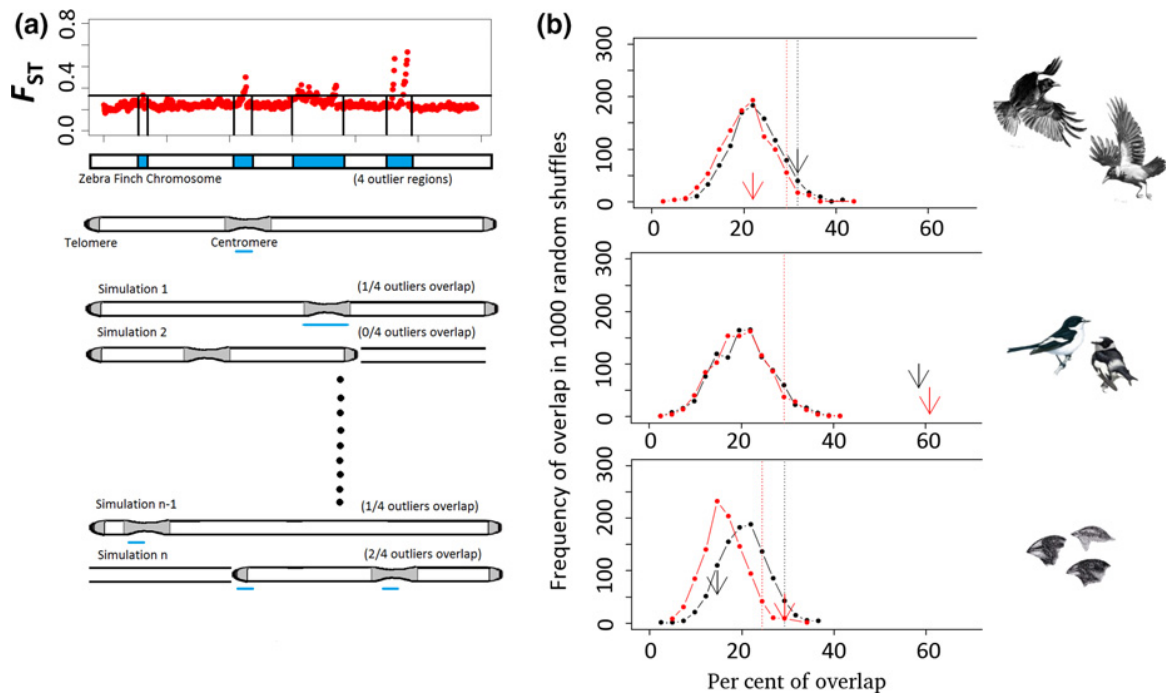

FIGURE 3 Association of genomic differentiation landscapes with chromosomal features. (a) Schematic of the shuffling of centromere and subtelomere positions to estimate the expectation for random overlap. (b) The degree of overlap between regions of elevated differentiation with the combined set of regions adjacent to the centro- and subtelomeres is quantified for two selected population pairs (red and black arrows) from each taxon. The distributions of random expectation as assessed by permutation for these population pairs are shown in the same colours. The dotted line to the right side is the $95 \%$ quantile of the distribution [Colour figure can be viewed at wileyonlinelibrary.com] 
ancestry, gene flow or common environmental factors can be excluded. Summary statistics capturing information on $\mathrm{N}_{\mathrm{e}}$ were correlated among clades spanning over 50 millions of years of divergence. The degree of correlation among clades was remarkable considering divergence times of several million generations, gaps in syntenic alignments and the statistical error associated with population genetic estimates from moderate samples sizes. With recombination rate being the key mediator of linked selection, an explanation of genomic parallelism in $N_{e}$ through linked selection requires conserved recombination landscapes among the clades under investigation. Unlike mammals, a relatively stable karyotype in birds (Ellegren, 2010) argues for global conservation of recombination landscape; however, the extent of such conservation is not clear, in particular at the level of individual chromosomes. Comparative analysis among chicken, zebra finch and collared flycatcher suggests that intrachromosomal rearrangements occurred at non-negligible rates and that lack of recombination around (macro-)chromosome centres appears to be specific to zebra finch (Kawakami et al., 2014). It is thus not straightforward to predict the degree of covariation in recombination rates at kb-resolution considered here. The observed correlation in population-scaled recombination rates between clades, however, is consistent with the assumption that overall recombination landscapes are sufficiently similar to mediate common patterns of linked selection. Nevertheless, it has been suggested that recombination rate could slightly change even within clades in birds (Kawakami et al., 2017), indicating that genetic diversity and differentiation could evolve in a species or clade-specific manner. It should further be noted that mutation rate variation could also contribute to the correlation However, compared to the effect of recombination rate, its effect on genomewide variation of genetic diversity seems minor (Cutter \& Payseur, 2013; Dutoit et al., 2017).

The magnitude of correlations of all summary statistics was not related to divergence time (Fig. S4) with sometimes noticeably higher correlation coefficients for the phylogenetically older flycatchercrow comparison, than for the younger flycatcher-finch comparison (Table S7). This suggests that the strength of covariation may be underestimated by factors such as genome quality, population sampling and/or differences in the degree of rearrangements between clades. Due to these limitations, a direct comparison of effect sizes between intra- and interclade comparisons which would allow the separation of population-specific selection from selection shared across all clades under consideration is at present not possible. However, substantial covariation among clades indicates that genomic regions with properties amenable to linked selection reducing $N_{e}$ remained stable across millions of years of evolution. The observation that $d_{x y}$ was generally reduced in areas of high relative differentiation $\left(F_{\mathrm{ST}}, \mathrm{PBS}\right)$ both within and across clades points towards a selective process continuously purging diversity and reducing effective population size (Cruickshank \& Hahn, 2014). Van Doren et al. (2017) also reported covariation in $F_{\mathrm{ST}}, d_{\mathrm{xy}}$ and $\pi$ across the shorter evolutionary distance between flycatchers and stonechat, and similarly concluded that linked selection continuously erodes local genetic diversity possibly before the divergence of these species.
Linked selection can occur in the form of background selection (Charlesworth, 1994) or recurrent hitch-hiking dynamics by selective sweeps (Smith \& Haigh, 1974). Consistent with both types of selection, recent population genetic studies of flycatchers and crows suggest that diversity and differentiation landscapes were associated with variation in recombination rate and gene density (as a proxy for the target of selection) within clades (Burri et al., 2015; Vijay et al., 2016). In species with moderate effective population sizes, beneficial mutations are expected to be limited, and the distribution of fitness effects are likely to differ between species (Eyre-Walker \& Keightley, 2007). Parallel positive selection forming the basis of adaptation or divergent selection affecting the same genomic regions in different clades is thus expected to be rare. Background selection on the other hand appears to be less limited by mutational input, assuming that the vast majority of new mutations are deleterious. Given its long-term effects, it will also be only slightly affected by the transitory population-specific demographic change (Beissinger et al., 2016; Coop, 2016; Ewing \& Jensen, 2016). Based on model-based coalescent simulation, Corbett-Detig, Hartl, and Sackton (2015) suggested that for species with low/moderate population sizes (including flycatchers), background selection would prevail over hitch-hiking in relative importance (but see Coop (2016) and Munch, Nam, Schierup, and Mailund (2016)). Importantly, linked selection based on either background selection or selective sweeps will reduce ancestral genetic variation and consequently generate shared patterns of reduced genetic diversity in low recombination regions. The observed negative correlation between $F_{\mathrm{ST}}$ and $d_{\mathrm{xy}}$ is consistent with predictions of linked selection of both background and positive selection reducing not only population-specific, but ancestral genetic variation. Yet, it cannot fully be excluded that loci directly governing population-specific adaptation or promoting population divergence can emerge in parallel among clades. Such an explanation would, however, need to invoke continuous and frequent occurrences of selective sweeps reducing genetic variation at syntenic regions between clades. The inclusion of more species from larger evolutionary distances with distinct biogeographic histories will help to further resolve the relative contribution of factors influencing local genetic diversity.

In all clades under investigation, we found evidence for reduced diversity and elevated differentiation at candidate (peri)centromeric regions. A similar association was suggested for mouse (Carneiro, Nuno, \& Nachman, 2009), Swainson's thrushes (Delmore et al., 2015) and stickleback fish (Roesti, Moser, \& Berner, 2013). These studies are consistent with the idea that strongly reduced recombination rate in the vicinity of centromeres will most strongly be affected by linked selection. However, centromeric positions in crow, flycatcher and Darwin's finch were approximated relative to centromeres in zebra finch. Zebra finch is known for its many lineage-specific inversions (Kawakami et al., 2014; Weissensteiner et al., 2017) which may have reduced the association of genetic differentiation with the predicted centromere locations in the target species. Recent work in crows, however, corroborates an impact of independently predicted, putative (peri)centromeric regions on population recombination, genetic 
diversity and differentiation (Weissensteiner et al., 2017). In addition to putative centromeric regions, we found evidence for an association of subtelomeric regions with variation in genetic diversity. Yet, subtelomeric regions are not necessarily characterized by low recombination in birds (Backström et al., 2010; Kawakami et al., 2014) which is consistent with an explanation invoking recurrent positive selection rather than background selection reducing local $N_{\mathrm{e}}$. However, in other systems, it has been shown that subtelomeric regions experience low recombination rates, similar to centromeres (Roesti et al., 2013). Further evaluation of this hypothesis will require fine-scale recombination rate estimates across all clades.

In conclusion, we advocate the use of comparative, phylogenetic approaches to shed light on population-level processes introducing heterogeneity in patterns of diversity, differentiation and divergence along the genome. Most insight will be gained in taxa with high-quality, chromosome level genome assemblies with correct placement of centromeric and subtelomeric regions. Independent estimates of mutation and recombination rates are further crucial to assess the genomic stability of these central processes across evolutionary timescales. On the bioinformatic side, unbiased methods for translating orthologous genomic coordinates among a large number of distantly related species are required.

\section{ACKNOWLEDGEMENTS}

Funding for this study was provided by the Swedish Research Council (grant number 621-2010-5553 to J.W., 2014-6325 to T.K. and 2013-08721 to H.E.), Marie Sklodowska Curie Actions (grant number 600398 to T.K.), the European Research Council (grant number ERCStG-336536 to J.W.), the Knut and Alice Wallenberg Foundation (to H.E. and J. W.) and the Swiss National Science Foundation (grants number PBLAP3-134299 and PBLAP3_140171 to R.B.). We are grateful for the access to the computational infrastructure provided by the UPPMAX Next-Generation Sequencing Cluster and Storage (UPPNEX) project, funded by the Knut and Alice Wallenberg Foundation and the Swedish National Infrastructure for Computing. We would like to thank Leif Andersson and his group for providing access to the genotype data from Lamichhaney et al. (2015). We are also grateful to Claire Peart for valuable input on the manuscript.

\section{DATA ACCESSIBILITY}

Raw data forming the basis for this study are publicly available at PRJNA192205 \& PRJEB9057 (Crows), PRJEB2984 (Flycatchers), PRJNA301892 (Darwin's Finches).

\section{AUTHOR CONTRIBUTIONS}

N.V. and J.W. conceived the study; N.V. conducted all bioinformatic analyses with help from M.W. R.B., T.K. and H.E. provided population genetic summary statistics for the flycatcher. N.V. and J.W. wrote the manuscript with input from all other authors.

\section{REFERENCES}

Abbott, R., Albach, D., Ansell, S., Arntzen, J. W., Baird, S. J. E., Bierne, N., ... Zinner, D. (2013). Hybridization and speciation. Journal of Evolutionary Biology, 26, 229-246.

Auton, A., Adi, F. A., Pfeifer, S., Venn, O., Ségurel, L., Street, T., ... McVean, G. (2012). A fine-scale chimpanzee genetic map from population sequencing. Science, 336, 193-198.

Backström, N., Forstmeier, W., Schielzeth, H., Mellenius, H., Nam, K., Bolund, E., ... Ellegren, H. (2010). The recombination landscape of the zebra finch Taeniopygia Guttata genome. Genome Research, 20, 485495.

Bank, C., Ewing, G. B., Ferrer-Admettla, A., Foll, M., \& Jensen, J. D. (2014). Thinking too positive? Revisiting current methods of population genetic selection inference. Trends in Genetics, 30, 540-546.

Barton, N. H. (1983). Multilocus clines. Evolution, 37, 454 471.

Barton, N. H., \& Bengtsson, B. O. (1986). The barrier to genetic exchange between hybridising populations. Heredity, 57, 357-376.

Beissinger, T. M., Wang, L., Crossby, K., Durvasula, A., Hufford, M. B., \& Ross-Ibarra, J. (2016). Recent demography drives changes in linked selection across the maize genome. Nature Plants, 2, 16084.

Brommer, J. E., Gustafsson, L., Pietiäinen, H., \& Merilä, J. (2004). Singlegeneration estimates of individual fitness as proxies for long-term genetic contribution. The American Naturalist, 163, 505-517.

Burri, R., Nater, A., Kawakami, T., Mugal, C. F., Olason, P. I., Smeds, L., ... Ellegren, $\mathrm{H}$. (2015). Linked selection and recombination rate variation drive the evolution of the genomic landscape of differentiation across the speciation continuum of Ficedula flycatchers. Genome Research, $25,1656-1665$.

Carneiro, M., Albert, F. W., Afonso, S., Pereira, R. J., Burbano, H., Campos, R., ... Ferrand, N. (2014). The genomic architecture of population divergence between subspecies of the European rabbit. PLOS Genetics, 10, e1003519.

Carneiro, M., Nuno, F., \& Nachman, M. W. (2009). Recombination and speciation: Loci near centromeres are more differentiated than loci near telomeres between subspecies of the European rabbit (Oryctolagus cuniculus). Genetics, 181, 593-606.

Chan, A. H., Jenkins, P. A., \& Song, Y. S. (2012). Genome-wide fine-scale recombination rate variation in Drosophila melanogaster. PLOS Genetics, 8, e1003090.

Charlesworth, B. (1994). The effect of background selection against deleterious mutations on weakly selected, linked variants. Genetical Research, 63, 213-227.

Charlesworth, B. (1998). Measures of divergence between populations and the effect of forces that reduce variability. Molecular Biology and Evolution, 15, 538-543.

Charlesworth, B., Morgan, M. T., \& Charlesworth, D. (1993). The effect of deleterious mutations on neutral molecular variation. Genetics, 134, 1289-1303.

Coop, G. (2016). Does linked selection explain the narrow range of genetic diversity across species?. bioRxiv, 042598. https://doi.org/10. 1101/042598

Corbett-Detig, R. B., Hartl, D. L., \& Sackton, T. B. (2015). Natural selection constrains neutral diversity across a wide range of species. PLoS Biology, 13, e1002112.

Cruickshank, T. E., \& Hahn, M. W. (2014). Reanalysis suggests that genomic islands of speciation are due to reduced diversity, not reduced gene flow. Molecular Ecology, 23, 3133-3157.

Cutter, A. D., \& Payseur, B. A. (2013). Genomic signatures of selection at linked sites: Unifying the disparity among species. Nature Reviews. Genetics, 14, 262-274.

Delmore, K. E., Hübner, S., Kane, N. C., Schuster, R., Andrew, R. L., Câmara, F., ... Irwin, D. E. (2015). Genomic analysis of a migratory divide reveals candidate genes for migration and implicates selective 
sweeps in generating Islands of differentiation. Molecular Ecology, 24, 1873-1888.

DePristo, M. A., Banks, E., Poplin, R., Garimella, K. V., Maguire, J. R., Hartl, C., ... Daly, M. J. (2011). A framework for variation discovery and genotyping using next-generation DNA sequencing data. Nature Genetics, 43, 491-498.

Dettman, J. R., Sirjusingh, C., Kohn, L. M., \& Anderson, J. B. (2007). Incipient speciation by divergent adaptation and antagonistic Epistasis in yeast. Nature, 447, 585-588.

Dutoit, L., Vijay, N., Mugal, C. F., Bossu, C. M., Burri, R., Wolf, J., \& Ellegren, $H$. (2017). Covariation in levels of nucleotide diversity in homologous regions of the avian genome long after completion of lineage sorting. Proceedings of the Royal Society. Series B, 284, 20162756.

Ellegren, H. (2010). Evolutionary stasis: The stable chromosomes of birds. Trends in Ecology \& Evolution, 25, 283-291.

Ellegren, H., \& Galtier, N. (2016). Determinants of genetic diversity. Nature Reviews Genetics, 17, 422-433.

Ellegren, H., Smeds, L., Burri, R., Olason, P. I., Backström, N., Kawakami, T., ... Wolf, J. B. (2012). The genomic landscape of species divergence in Ficedula Flycatchers. Nature, 491, 756-760.

Ericson, P. G. P., Zuccon, D., Ohlson, J. I., Johansson, U. S., Alvarenga, H., \& Prum, R. O. (2006). Higher-level phylogeny and morphological evolution of Tyrant Flycatchers, Cotingas, Manakins, and Their allies (Aves: Tyrannida). Molecular Phylogenetics and Evolution, 40, 471-483.

Ewing, G. B., \& Jensen, J. D. (2016). The consequences of not accounting for background selection in demographic inference. Molecular Ecology, 25, 135-141.

Eyre-Walker, A., \& Keightley, P. D. (2007). The distribution of fitness effects of new mutations. Nature Reviews Genetics, 8, 610-618.

Gompert, Z., \& Buerkle, C. A. (2011). Bayesian estimation of genomic clines. Molecular Ecology, 20, 2111-2127.

Goudet, J. (2005). Hierfstat, a package for R to compute and test hierarchical F-statistics. Molecular Ecology Notes, 5, 184-186.

Grant, P. R., \& Grant, B. R. (1992). Hybridization of bird species. Science, 256, 193-197.

Harris, R. S. (2007). Improved Pairwise Alignment of Genomic DNA. Phd thesis, Pennsylvania State University.

Hinrichs, A. S., Karolchik, D., Baertsch, R., Barber, G. P., Bejerano, G., Clawson, H., ... Kent, W. J. (2006). The UCSC genome browser database: Update (2006). Nucleic Acids Research, 34, D590-D598.

Hodgkinson, A., \& Eyre-Walker, A. (2011). Variation in the mutation rate across mammalian genomes. Nature Reviews Genetics, 12, 756-766.

Hooper, D. M., \& Price, T. D. (2015). Rates of karyotypic evolution in Estrildid finches differ between island and continental clades. Evolution, 69, 890-903.

Hubbard, T. D., Barker, D., Birney, B. E., Cameron, G., Chen, Y., Clark, L., \& ... Clamp, M. (2002). The Ensembl genome database project. Nucleic Acids Research, 30, 38-41.

Hudson, R. R., \& Coyne, J. A. (2002). Mathematical consequences of the genealogical species concept. Evolution, 56, 1557-1565.

Jarvis, E. D., Mirarab, S., Aberer, A. J., Li, B., Houde, P., Li, C., ... Zhang, G. (2014). Whole-genome analyses resolve early branches in the tree of life of modern birds. Science, 346, 1320-1331.

Jetz, W., Thomas, G. H., Joy, J. B., Hartmann, K., \& Mooers, A. O. (2012). The global diversity of birds in space and time. Nature, 491, 444 448.

Jetz, W., Thomas, G. H., Joy, J. B., Redding, D. W., Hartmann, K., \& Mooers, A. O. (2014). Global distribution and conservation of evolutionary distinctness in birds. Current Biology, 24, 919-930.

Jønsson, K. A., Fabrea, P. H., Kennedy, J. D., Holt, B. G., Borregaard, M. K., Rahbek, C., \& Fjeldså, J. (2016). A supermatrix phylogeny of Corvoid Passerine Birds (Aves: Corvides). Molecular Phylogenetics and Evolution, 94, Part A: 87-94.

Kawakami, T., Mugal, C. F., Suh, A., Nater, A., Burri, R., Smeds, L., \& Ellegren, H. (2017). Whole-genome patterns of linkage disequilibrium across flycatcher populations clarify the causes and consequences of fine-scale recombination rate variation in Birds. Molecular Ecology, https://doi.org/10.1111/mec.14197.

Kawakami, T., Smeds, L., Backström, N., Husby, A., Qvarnström, A., Mugal, C. F., ... Ellegren, H. (2014). A high-density linkage map enables a second-generation collared flycatcher genome assembly and reveals the patterns of avian recombination rate variation and chromosomal evolution. Molecular Ecology, 23, 4035-4058.

Knief, U., \& Forstmeier, W. (2016). Mapping centromeres of microchromosomes in the zebra finch (Taeniopygia Guttata) using half-tetrad analysis. Chromosoma, 125, 757-768.

Kronforst, M. R., \& Papa, R. (2015). The functional basis of wing patterning in Heliconius butterflies: The molecules behind mimicry. Genetics, 200, 1-19.

Kuhn, R. M., Karolchik, D., Zweig, A. S., Trumbower, H., Thomas, D. J., Thakkapallayil, A., ... Kent, W. J. (2007). The UCSC genome browser database: Update 2007. Nucleic Acids Research, 35, D668-D673.

Lamichhaney, S., Berglund, J., Almén, M. S., Maqbool, K., Grabherr, M., Martinez-Barrio, A., ... Andersson, L. (2015). Evolution of Darwin's finches and their beaks revealed by genome sequencing. Nature, 518, 371-375.

Lewontin, R. C., \& Krakauer, J. (1973). Distribution of gene frequency as a test of the theory of the selective neutrality of polymorphisms. Genetics, 74, 175-195.

Mallet, J., Beltrán, M., Neukirchen, W., \& Linares, M. (2007). Natural hybridization in Heliconiine butterflies: The species boundary as a continuum. BMC Evolutionary Biology, 7, 28.

Munch, K., Nam, K., Schierup, M. H., \& Mailund, T. (2016). Selective sweeps across twenty millions years of primate evolution. Molecular Biology and Evolution, 33, 3065-3074.

Nachman, M. W., \& Payseur, B. A. (2012). Recombination rate variation and speciation: Theoretical predictions and empirical results from rabbits and mice. Philosophical Transactions of the Royal Society of London Series B, Biological Sciences, 367, 409-421.

Nadachowska-Brzyska, K., Burri, R., Olason, P. I., Kawakami, T., Smeds, L., \& Ellegren, H. (2013). Demographic divergence history of Pied Flycatcher and Collared Flycatcher inferred from whole-genome resequencing data. PLOS Genetics, 9, e1003942.

Nei, M. (1987). Molecular evolutionary genetics (equation 10.20). New York City, NY: Columbia University Press.

Nosil, P., \& Feder, J. L. (2013). Genome evolution and speciation: Toward quantitative descriptions of pattern and process. Evolution, 67, 24612467.

Poelstra, J. W., Vijay, N., Bossu, C. M., Lantz, H., Ryll, B., Müller, I., .. . Wolf, J. B. W. (2014). The genomic landscape underlying phenotypic integrity in the face of gene flow in Crows. Science, 344, 1410 1414.

Poelstra, J. W., Vijay, N., Hoeppner, M. P., \& Wolf, J. B. (2015). Transcriptomics of colour patterning and coloration shifts in crows. Molecular Ecology, 24, 4617-4628.

Powell, T. H. Q., Hood, G. R., Murphy, M. O., Heilveil, J. S., Berlocher, S. H., Nosil, P., \& Feder, J. L. (2013). Genetic divergence along the speciation continuum: The transition from host race to species in Rhagoletis (Diptera: Tephritidae). Evolution, 67, 2561-2576.

Prum, R. O., Berv, J. S., Dornburg, A., Field, D. J., Townsend, J. P., Lemmon, E. M., \& Lemmon, A. R. (2015). A comprehensive phylogeny of birds (Aves) using targeted next-generation DNA sequencing. Nature, 526, 569-573.

Puzey, J. R., Willis, J. H., \& Kelly, J. K. (2017). Population structure and local selection yield high genomic variation in Mimulus guttatus. Molecular Ecology, 26, 519-535.

Quinlan, A. R., \& Hall, I. M. (2010). BEDTools: A flexible suite of utilities for comparing genomic features. Bioinformatics, 26, 841-842.

Rands, C. M., Darling, A., Fujita, M., Kong, L., Webster, M. T., Clabaut, C., ... Ponting, C. P. (2013). Insights into the evolution of Darwin's 
Finches from comparative analysis of the Geospiza Magnirostris genome sequence. BMC Genomics, 14, 95.

Renaut, S., Grassa, C. J., Yeaman, S., Moyers, B. T., Lai, Z., Kane, N. C., ... Rieseberg, L. H. (2013). Genomic Islands of divergence are not affected by geography of speciation in sunflowers. Nature Communications, 4, 1827.

Roesti, M., Hendry, A. P., Salzburger, W., \& Berner, D. (2012). Genome divergence during evolutionary diversification as revealed in replicate Lake-stream Stickleback population pairs. Molecular Ecology, 21, 2852-2862.

Roesti, M., Kueng, B., Moser, D., \& Berner, D. (2015). The genomics of ecological vicariance in Threespine Stickleback Fish. Nature Communications, 6, 8767.

Roesti, M., Moser, S., \& Berner, D. (2013). Recombination in the threespine stickleback genome-patterns and consequences. Molecular Ecology, 22, 3014-3027

Romanov, M. N., Farré, M., Lithgow, P. E., Fowler, K. E., Skinner, B. M., O'Connor, R., ... Griffin, D. K. (2014). Reconstruction of gross Avian genome structure, organization and evolution suggests that the Chicken lineage most closely resembles the Dinosaur Avian ancestor. BMC Genomics, 15, 1060.

Scheet, P., \& Stephens, M. (2006). A fast and flexible statistical model for large-scale population genotype data: Applications to inferring missing genotypes and haplotypic phase. American Journal of Human Genetics, 78, 629-644.

Seehausen, O., Butlin, R. K., Keller, I., Wagner, C. E., Boughman, J. W., Hohenlohe, P .A., ... Widmer, A. (2014). Genomics and the origin of species. Nature Reviews Genetics, 15, 176-192.

Shafer, A. B. A., Peart, C. R., Tusso, S., Maayan, I., Brelsford, A., Wheat, C. W., \& Wolf, J. B. W. (2016). Bioinformatic processing of RAD-Seq data dramatically impacts downstream population genetic inference. Methods in Ecology and Evolution. online early, https://doi.org/10. 1111/2041-210X.12700.

Singhal, S., Leffler, E. M., Sannareddy, K., Turner, I., Venn, O., Hoope, D. M., ... Przeworski, M. (2015). Stable recombination hotspots in birds. Science, 350, 928-932.

Slotte, T. (2014). The impact of linked selection on plant genomic variation. Briefings in Functional Genomics, 13, 268-275.

Smith, T., \& Eyre-Walker, A. (2017). Large scale variation in the rate of de novo mutation in humans and its relationship to divergence and diversity. bioRxiv, 110452. https://doi.org/10.1101/110452

Smith, J. M., \& Haigh, J. (1974). The hitch-hiking effect of a favourable gene. Genetical Research, 23, 23-35.

Soria-Carrasco, V., Gompert, Z., Comeault, A. A., Farkas, T. E., Parchman, T. L., Johnston, J. S., ... Nosil, P. (2014). Stick insect genomes reveal natural selection's role in parallel speciation. Science, 344 738-742.

Stephan, W. (2010). Genetic hitchhiking versus background selection: The controversy and its implications. Philosophical Transactions of the Royal Society of London B: Biological Sciences, 365, 1245-1253.

Strasburg, J. L., Sherman, N. A., Wright, K. M., Moyle, L. C., Willis, J. H., \& Rieseberg, L. H. (2012). What can patterns of differentiation across plant genomes tell us about adaptation and speciation? Philosophical Transactions of the Royal Society of London B: Biological Sciences, 367, 364-373.

Tang, H., Li, J., \& Krishnakumar, V. (2015). Jcvi: JCVI Utility Libraries.
Tine, M., Kuhl, H., Gagnaire, P. A., Louro, B., Desmarais, E., Martins, R. S. T., \& Reinhardt, R. (2014). European sea bass genome and its variation provide insights into adaptation to Euryhalinity and speciation. Nature Communications, 5, 5770.

Van Doren, B. M., Campagna, L., Helm, B., Illera, J. C., Lovette, I. J., \& Liedvogel, M. (2017). Correlated patterns of genetic diversity and differentiation across an Avian family. Molecular Ecology. https://doi. org/10.1111/mec.14083.

Vijay, N., Bossu, C. M., Poelstra, J. W., Weissensteiner, M. H., Suh, A., Kryukov, A. P., \& Wolf, J. B. W. (2016). Evolution of heterogeneous genome differentiation across multiple contact zones in a crow species complex. Nature Communications, 7, 13195.

Vijay, N., Poelstra, J. W., Künstner, A., \& Wolf, J. B. (2013). Challenges and strategies in transcriptome assembly and differential gene expression quantification. a comprehensive in silico assessment of RNA-Seq experiments. Molecular Ecology, 22, 620-634.

Weber, C. C., Boussau, B., Romiguier, J., Jarvis, E. D., \& Ellegren, H. (2014). Evidence for GC-Biased gene conversion as a driver of between-lineage differences in Avian base composition. Genome Biology, 15, 549.

Weissensteiner, M. H., Pang, A. W. C., Bunikis, I., Höijer, I., Vinnere-Petterson, O., Suh, A., \& Wolf, J. B. W. (2017). Combination of shortread, long-read and optical mapping assemblies reveals presumably heterochromatic tandem repeat arrays with population genetic implications. Genome Research, 27, 697-708.

Wolf, J. B. W., Bayer, T., Haubold, B., Schilhabel, M., Rosenstiel, P., \& Tautz, D. (2010). Nucleotide divergence vs. gene expression differentiation: Comparative transcriptome sequencing in natural isolates from the carrion crow and its hybrid zone with the hooded crow. Molecular Ecology, 19, 162-175.

Wolf, J. B. W., \& Ellegren, H. (2017). Making sense of genomic islands of differentiation in light of speciation. Nature Reviews Genetics, 18, 87 100.

Wolf, J. B. W., Lindell, J., \& Backström, N. (2010). Speciation genetics: Current status and evolving approaches. Philosophical Transactions of the Royal Society B: Biological Sciences, 365, 1717-1733.

$\mathrm{Wu}, \mathrm{C}$. I. (2001). The genic view of the process of speciation. Journal of Evolutionary Biology, 14, 851-865.

\section{SUPPORTING INFORMATION}

Additional Supporting Information may be found online in the supporting information tab for this article.

How to cite this article: Vijay N, Weissensteiner M, Burri R, Kawakami T, Ellegren H, Wolf JBW. Genomewide patterns of variation in genetic diversity are shared among populations, species and higher-order taxa. Mol Ecol. 2017;26:4284 4295. https://doi.org/10.1111/mec.14195 